\title{
DER DEUTSCHE REPUBLIKANER JOHANN PHILIPP BECKER ALS MITSTREITER FÜR DIE ERRINGUNG DEMOKRATISCHER ZIELE IN DER SCHWEIZ
}

EIN BEITRAG ZUR GESCHICHTE DES VORMÄRZ IM KANTON BERN

„Mein Freund, Joh. Phil. Becker zu Biel, steht im Begriff gleich mir, ein Bürgerrecht im Kanton Bern nachzusuchen. Könnten Sie ihm dabei behilflich sein, so bitte ich Sie, es zu thun. Ich kenne keinen tüchtigeren Mann, an ihm wird der Kanton einen thätigen, kräftigen und hochherzigen Bürger gewinnen. Nach meiner Überzeugung wiegen alle Deutsche, die seit 3 Jahren Bürger in Bern geworden, diesen einen nicht auf."1 So schrieb Karl Mathy im Januar 1842 in einem Brief an Regierungstath Dr. J. R. Schneider.

Wer war dieser Becker, für den sich Mathy so einsetzte? Becker wurde am 20.3.1809 in Frankenthal in der Pfalz als Sohn eines Handwerkers geboren. Wie aus der französisch geschriebenen Geburtsurkunde hervorgeht, erhielt er den Vornamen Jean Philippe. ${ }^{2}$ Da die Pfalz bis I 8 I 4 französisch war, blieb Becker bis dahin französischer citoyen. Die Eindrücke der Jugend wirken oft bestimmend auf das spätere Leben der Menschen ein. So war es auch bei dem jungen Becker. Er nahm den fortschrittlichen Geist, den die Französische Revolution in seine Heimat getragen hatte, schon als Junge in sich auf und blieb den Ideen der Großen Bürgerlichen Französischen Revolution, „liberté, égalité, fraternitê", sein ganzes Leben lang treu.

Diese Ideen aber mußten Becker bald mit den bestehenden $\mathrm{Zu}$ ständen in seiner 1816 bayrisch gewordenen Heimat in Konflikt bringen. Somit wurde Becker schon in seiner frühen Jugend ein „unbestimmter Oppositionsmann". ${ }^{3}$

Als sich 1832 in Hambach die fortschrittlichsten Kräfte des damaligen Deutschlands versammelten, da gehörte Becker trotz seiner Jugend schon zu denen, die auf der Schloßruine als Redner auf-

1 Karl Mathy, Brief aus Karlsruhe vom i 5./30.1.1842, Nr. 41 an Regierungsrath Dr. J. R. Schneider, in: Nachlaß Dr. J. Schneiders, Korrespondenz L-S, in: Staatsarchiv Bern.

2 Geburtsurkunde Beckers, Nr. 26, 1809, Frankenthal, Archiv.

${ }^{3}$ F. Engels, Dem Gedächtnis Johann Philipp Beckers, in: Der Sozialdemokrat, Organ der Sozialdemokratie deutscher Zunge, Zürich vom 17.1 2.1 886, Nr. s I, Titelseite. 
traten. ${ }^{1}$ In seinen Ausführungen forderte er, daß sich die Menschen zu einer Organisation zusammenschließen und sich bewaffnen sollten, um die Fürsten von den Thronen zu stürzen.

Es ist von besonderer Bedeutung, daß Becker in Hambach mit den fortschrittlichen Vertretern der damaligen deutschen Intelligenz, mit Dr. Siebenpfeiffer und Dr. Wirth, zusammentraf. Beide Männer haben das politische Bewußtsein des Pfälzers mit geformt und dazu beigetragen, daß weder Verhaftungen noch Verfolgungen ihn von dem einmal eingeschlagenen Wege abbringen konnten. Als Becker wegen seines Auftretens in Hambach verhaftet wurde und danach den Kampf in Deutschland nicht mehr fortsetzen konnte, emigrierte er 1838 in die Schweiz.

In seiner Wahlheimat, der Schweiz, hat Becker fast so Jahre lang - bis zu seinem Tode I 886 - aktiv am politischen Geschehen teilgenommen. Während er später einer der rührigsten Kämpfer der Internationalen Arbeiterassoziation (IAA) wurde, beteiligte er sich während der ersten Jahre seines Schweizer Aufenthaltes aktiv an den vormärzlichen Bestrebungen der Schweizer Demokratie. Diesem Zeitabschnitt soll folgender Artikel gewidmet sein.

\section{BECKERS ÜBERSIEDLUNG IN DIE SCHWEIZ, ZUNÄCHST NACH BERN}

Becker hatte schon im Jahre 1837 Reisen in die Schweiz unternommen, um sich zu orientieren, wo er im Falle einer Übersiedlung in die Schweiz eine neue Heimstätte finden könne. Am 27.5. I 838 wurde ihm das Recht erteilt, sich mit seiner Familie in Bern niederzulassen. ${ }^{2}$ Die Emigration in die Schweiz war vor allem auf Anraten von Beckers

1 J. Ph. Becker, Seine Rede in Hambach, abgedruckt in Wirth J. G. H.: Das Nationalfest der Deutschen zu Hambach, Neustadt a. H. r832, S. 85-87, außerdem in: Monatsschrift des Frankenthaler Altertumsvereins, Frankenthal 1932, 40. Jahrg., Nr. 5 S. I7-18. Vergleiche dazu auch: ders., Etwas über das Hambacher Fest, in: Der arme Conrad, Illustrierter Kalender für das arbeitende Volk, Leipzig 1876, I. Jg., S. 34-36; ders., Offener Brief an die deutschen Parteigenossen bei Gelegenheit der 50 jährigen Gedenkfeier des Hambacher Festes, Genf 27.5.1882, Zürich 1882; Vollständige Verhandlungen vor dem Kgl. Bayr. Appellationsgerichte des Rheinkreises und in den öffentlichen Sitzungen des außerordentlichen Assisengerichts zu Landau vom 29.7.1833 und der folgenden Tage gegen Dr. Wirth, Dr. Siebenpfeiffer, Hochdörfer, Scharpff, Becker, Dr. Grosse, Dr. Pistor, Rost und Baumann. Hrg. von Ludwig Hoffmann, Zweibrücken 1833 , S. $30 \mathrm{ff}$.

2 Manual des Regierungsrates, Nr. 6 vom 15. Juni 1838-28. Juli 1838, Seite 539, in: Staatsarchiv Bern. Bewilligung für Herrn Joh. Phil. Becker aus Bayern, um sich als Bürstenfabrikant mit seiner Familie in Bern niederzulassen, gegen Deposition eines gehörig legalisierten, für 2 Jahre gültigen Heimatscheins vom 21 . März I 836. 
Freunden, Karl Mathy und Dr. Siebenpfeiffer, erfolgt. ${ }^{1}$ Dr. Siebenpfeiffer lehrte damals als Professor an der Universität Bern. Während seines kurzen Berner Aufenthaltes beteiligte sich Becker von der Aarestadt aus am sogenannten „Prinzenhandel”. Die französischen Drohungen hatten die in ihrem Nationalgefühl verletzten Schweizer veranlaßt, Verteidigungsmaßnahmen einzuleiten. Während in einer Linie von Genf bis Basel zwei Schweizer Korps die Grenze decken sollten, beteiligten sich auch Schützenvereine und Freikorps an dieser Aktion. ${ }^{2}$ Bei der Errichtung eines solchen Freikorps war Becker mit tätig. ${ }^{3}$ Verständlich wird uns seine Handlungsweise aus dem $\mathrm{Haß}$, den er damals gegen Frankreich hegte und der manchmal sogar chauvinistische Formen annahm. ${ }^{4}$ Becker sah in Frankreich und Rußland die beiden Hauptfeinde Deutschlands:

„Frankreich bedroht uns mit Verstümmelung und Entehrung durch Wegnahme des Rheins; Rußland lastet mit dem ganzen Gewicht seines absoluten Staatsprinzips auf unserer inneren Entwicklung und bedroht uns mit dem Verlust freier Benutzung der Donaumündungen; in Frankreich wird die bürgerliche Jugend erhitzt mit der Idee eines Weltreiches; in Rußland wird die adlige Jugend mit gleichen Gedanken gestachelt." 5

Natürlich wurde Beckers Eintreten für Louis Bonaparte 1838 auch von der allgemeinen Meinung, die damals über den französischen Thronprätendenten in der Schweiz herrschte, mitbestimmt. Gab es doch viele, die sich von dem jovialen und biederen Wesen des Prinzen täuschen ließen, der gerade in Bern eine „echt radikale Gesinnung” zur Schau trug. ${ }^{6}$

Wie dem auch sei, es erscheint mir als eine Ironie der Geschichte, daß Beckers erstes politisches Handeln in der Schweiz, in jenem Lande, in dem er einer der Vorkämpfer der internationalen Arbeiter-

1 Ehrerbietige Bitte an die hohe Regierung der Republik Bern von Joh. Phil. Becker, Kaufmann, Fabrikant aus Frankenthal in Rheinbayern, dermalen wohnend in Biel, sich in einem Orte des Kantons ein Bürgetrecht erwerben zu dürfen, in: Die Vorträge der Direktion der Justiz und Polizei, Mappe r.-10. Oktober 1846, in: Staatsarchiv Bern.

2 P. Feddersen, Geschichte der Schweizerischen Regeneration von I830-1848, Zürich I 867 , S. $272-273$.

${ }^{3}$ N. Rjasanoff, Zur Biographie von Johann Philipp Becker. Sein Curriculum vitae bis 1856, in: Archiv für die Geschichte des Sozialismus und der Arbeiterbewegung, herausgegeben von Carl Grünberg, Leipzig 1914, 4. Jg., S. 316.

${ }^{4} \mathrm{~J}$. Ph. Becker, Ein Wort über die Fragen der Zeit, seinen Mitbürgern zum Geschenk von Johann Philipp Becker, einem deutschen Bürger aus dem Gewerbestande, Bellevue bei Konstanz I 84 I, S. 32 .

5 Ebenda, S. 3 I.

- E. Blösch, Prinz Louis Napoleon in Bern. Berner Taschenbuch I881, S. 22 I-229. 
bewegung werden sollte, ausgerechnet eine Angelegenheit betraf, die zugunsten des Prinzen Napoleon bestimmt war, jenes Mannes, den Becker später, als Louis Bonaparte Kaiser der Franzosen geworden war, im „Vorboten” so heftig angriff, ${ }^{1}$ bei dessen Sturz Becker 1870 sarkastisch in seiner Zeitschrift bemerkte:

„Die Vorsehung der Gewalt der Umstände, die dich zeitweilig zum Napoleon III. erhoben, hat dich nun in ihrem hohen Ratschlusse wieder zum Alltagsmenschen, dem simplen Louis herabgedrückt. Und so stehst du nun wieder, wie s.Z. vor Straßburg und Boulogne, als erbarmungswürdiger Simplicius $\mathrm{da}$ - und ach, sogar, weil inzwischen verlorengegangen, ohne die Zauberkraft deines Namens. Doch ein Trost ist dir geblieben: daß die 'Große Nation' [sich der zwanzigjährigen Herrschaft deiner 'Kapazität'] mehr zu schämen hat, als Du dich der von der grausamen Natur dir so spärlich gespendeten Anlagen." 2

\section{BECKERS ÜBERSIEDLUNG NACH BIEL}

Da Becker in Bern einen unergiebigen Boden für seine industrielle Tätigkeit fand ${ }^{3}$, begab er sich am Anfang des Jahres I839 nach dem gewerbefleißigen Biel, jener landschaftlich so schön gelegenen Stadt, in deren Mauern und Umgebung schon oft freiheitlich gesinnte Menschen Zuflucht gesucht hatten. Eine Zeitlang lebte Galeer, der Führer des Schweizer Grütlivereins, hier. In dem nahegelegenen Bade Grenchen hatte sich Mazzini vor der Polizei verborgen. ${ }^{4}$

Vom Regierungsstatthalteramt Biel liegt eine vom 25.1.1839 datierte Niederlassungsbewilligung vor, die die Behörde auf ein Leumundszeugnis aus Bern vom 7.12.1838 ausgestellt hatte. Johann Philipp Becker wurde dann am 9.3.1839 in Biel zum „Einsassen” angenommen. ${ }^{5}$ Er wohnte zunächst mit seiner Familie im Hause des Herrn Lorenz Dietz an der Obergasse (heute Haus Nr. I3). ${ }^{6}$ Gewiß waren es anfangs sehr schwere Monate, die die Familie Becker in Biel verbrachte. Heimat und liebe Bekannte hatte man in Rhein-

1 J. Ph. Becker, in: Der Vorbote. Politische und sozialökonomische Zeitschrift. Zentralorgan der Sektionsgruppe deutscher Sprache der Internationalen Arbeiterassoziation, redigiert von J. Ph. Becker, Genf I 866-71, siehe: 1866, Heft 6, S. 83; 1867, Heft 4, S. 52 ; 1867, Heft 7, S. 102; 1867, Heft II, S. I64; 1868, Heft 6, S. 89-92.

2 Ebenda, Heft 8, 1870, S. 114 .

3 J. Ph. Becker, Ehrerbietige Bitte..., a.a.O.

4 E. Blösch, Eduard Blösch und 30 Jahre Bernischer Geschichte, Bern I872, S. 229.

5 Bürgerrodel II, Bürgerkanzlei Biel.

- Kontrolle über die etablierten und haushablichen Einsassen von Biel 1832-I847, Stadtarchiv Biel. (Für diese Mitteilung danke ich Hetrn Stadtarchivar Bourquin in Biel.) 
bayern zurückgelassen. ${ }^{1}$ Eine sehr unsichere Zukunft lag vor ihnen. Dazu kam noch, daß Frau Becker damals erneut in Hoffnung war. Frau Elisabeth Becker, geb. Seser, ertrug in vorbildlicher Weise, in Hingabe und Opferbereitschaft all das an Entbehrungen, was ihr das politische Wirken ihres Mannes auferlegte. Sie war selbst ein Kind der heiteren Pfalz und hat sicher nur schweren Herzens ihre Eltern- und Heimatstadt Frankenthal verlassen, jene Stadt, in der sie bis zur Emigration in die Schweiz schon 3 Kindern das Leben geschenkt hatte.

Am I 3.I0. I 827 war Gottfried geboren worden. Er hatte den ideellen Sinn und das unstete Blut des Vaters geerbt. ${ }^{2}$ Er wanderte später nach Amerika aus, beteiligte sich dort als Journalist und Redakteur, ${ }^{3}$ nahm an dem Sezessionskrieg teil, rückte an der Spitze eines Regiments gegen die südlichen Staaten ins Feld. Er wurde in zwei Schlachten schwer verwundet. Das Generalspatent erhielt er gerade in dem Augenblick, als er seinen Verletzungen erlag. ${ }^{4}$ In die Frankenthaler Zeit fällt auch die Geburt eines Mädchens (29.9.I83 I) und die Geburt von Georg Becker am 24.7.1834. Letzterer wurde ein ganz bedeutender Schweizer Musikschriftsteller. Seiner musikwissenschaftlichen Verdienste wegen wurde er zum Dr. h.c. der Genfer Universität promoviert. Er gab unter anderem das auch in Fachkreisen geschätzte Werk: „La musique en Suisse depuis les temps les plus reculés jusqu'à la fin du XVIIIe siècle" heraus. ${ }^{5}$ Er starb hochgeachtet 1928 in Genf. Auch in Biel riß der Kindersegen der Familie Becker nicht ab. Schon am 2.3.1839 erblickte Ernst Hermann das Licht der Welt. Er unterstützte seinen Vater später bei redaktionellen Arbeiten am „Vorboten”. Während der Jahre bis r 848 gebar Frau Becker noch drei Mädchen: Josephine, Elise, Johanna. Über das Schicksal von Beckers Kindern können wir ausführlicher in einem Brief Beckers an Sorge lesen. ${ }^{6}$

\footnotetext{
${ }^{1}$ Ferdinand Lingenau, Brief an Joh. Phil. Becker vom 21.12.1874 und 23.7.1875, in: Beckers Nachlaß, Internationales Institut für Sozialgeschichte, Amsterdam.

${ }^{2}$ Werner Bourquin, Schweizer Holz- und Bauarbeiterverband, 25 Jahre Sektion Biel, erschienen in Biel I955, S. 45.

${ }^{3}$ Carl Wittke, Refugees of Revolution. The German Forty-Eighters in America, Philadelphia I952, S. 229.

4 Reinhold Rüegg, Aus Briefen an Johann Philipp Becker, in: Die Neue Zeit. Revue des geistigen und öffentlichen Lebens. 6. Jahrgang, Stuttgart i 888, S. 45 x.

${ }^{5}$ Historisch-biographisches Lexikon der Schweiz, Band 2, Neuenburg 1924, S. 67.

${ }^{6} \mathrm{~J}$. Ph. Becker, Brief vom 3.8.1867 aus Genf an F. A. Sorge, in: Briefwechsel Beckers an Sorge 1867-1883, in: Schweizerisches Sozialarchiv Zürich. - Dieser bisher unveröffentlichte Brief, der mir freundlicherweise als Kopie von Herrn Dr. Eugen Steinemann vom Schweizerischen Sozialarchiv Zürich zur Verfügung gestellt wurde, gibt einen guten Einblick in Beckers Familienverhältnisse. Siehe auch: Bürgerrodel II, Bürgerkanzlei Biel.
} 
3. BECKERS BERUFLICHE TÄTIGKEIT IN BIEL

In Biel betrieb Becker zunächst gemeinschaftlich mit Herrn Ernst Schüler Weinhandel und ein Uhrengeschäft. Seit I 84I handelte er mit Zigarren, die er aus Bremen und Brüssel bezog und in der Schweiz vertrieb. Da dies ein sehr einträgliches Geschäft war, ging Becker I 842 dazu über, zusammen mit R. Moser selbst Zigarren herzustellen. Damit erwarb sich Becker das Verdienst, in Biel die Zigarrenherstellung eingeführt und eine neue Verdienstmöglichkeit in der Stadt geschaffen zu haben. Die Bieler Bevölkerung schätzte dies umso mehr, weil Erwerbslosigkeit seit dem 1842 erfolgten Eingehen der Indiennefabrik in Biel viele Arbeiter bedrohte. ${ }^{1}$

Beckers geschäftliche Unternehmungen gingen so gut, daß er 1846 die Arbeiterzahl in seinem Betrieb erneut erhöhen konnte. Er bezog damals aus den verschiedensten Teilen der Welt Tabak, so aus Holland, Westindien, Ostindien, Nordamerika, Brasilien, dem Elsaß und der Pfalz. Schließlich kaufte er mit Herrn Stadtforstverwalter Greyerz aus Biel ein Wasserwerk, teils um dadurch die immer mehr aufblühende Uhrenindustrie zu unterstützen, teils um dort Zigarrenkisten anfertigen zu lassen. ${ }^{2} \mathrm{DaB}$ Becker sich mit seiner schweizerischen Wahlheimat verbunden fühlte, dürfte aus der Tatsache entnommen werden, daß er am 26.10.1846 für 1 50 Louisdor als Bürger Biels aufgenommen wurde. ${ }^{3}$ Mit der Verbesserung seiner materiellen Lage hängt es auch zusammen, daß er 1846 das Haus Nr. 258 erwarb, das noch heute an der Obergasse steht und die Nummer 33 trägt. Auf den runden Befestigungsturm setzte Becker einen hölzernen Aufbau. In diesem Holzturm trocknete er seinen Tabak. Er führte außerdem im Hause eine Wirtschaft, die er später in Erinnerung an die Genfer Revolution I 846 "Café St. Gervais" nannte. ${ }^{4}$ Für seine wirtschaftlichen Erfolge in Biel erhielt er von der ökonomischen Gesellschaft sogar ein Diplom. ${ }^{5}$

1 Werner Bourquin, Schweizer Holz- und Bauarbeiterverband, a.a.O., S. 44.

2 J. Ph. Becker, Ehrerbietige Bitte..., a.a.O.

${ }^{3}$ Bürgerrodel II, Bürgerkanzlei Biel; vergl. auch das Protokoll des Großen Raths, Nr. 2 I vom 8.2.1846 bis 23.3.1847, S. $47 \mathrm{I}$ vom 25.1.1847, aus dem hervorgeht, daß Becker auch das Kantonsbürgerrecht erhielt. Darin heißt es: dem Herrn Johann Philipp Becker, gebürtig aus Frankenthal in Rheinbayern, reformierter Konfession, in Biel wohnhaft, dem die Bürgergemeinde die Zusicherung der Aufnahme in ihr Bürgerrecht erteilt hat. Abstimmung durch Ballotierung: für Willfahr 92 Stimmen, für Abschlag 39. Die erforderliche Stimmenzahl von 2 Drittel der Anwesenden betrug 88. Siehe auch: Manual des Regierungsrates, Nr. 122 vom Ir.1.1847 bis 18.2.1847, Schreiben vom 23.x.1847, S. 165 und vom 26.1.1847, S. 196; Die Vorträge der Direktion der Justiz und Polizei, Mappe 1.-10. Oktober 1846, Nr. 359, in: Staatsarchiv Bern.

4 Werner Bourquin, Schweizer Holz- und Bauarbeiterverband, a.a.O., S. 44.

5 Fernand Schwab, Die industrielle Entwicklung der Stadt Biel, Biel 1918 (Diss. Bern), S. 24-26. 
Obwohl Becker sich in Biel eine gesicherte Existenz aufgebaut hatte, die von staatlicher Seite auch entsprechend gewürdigt wurde, ${ }^{1}$ gab er sein altes Ideal, ein Kämpfer für die menschliche Freiheit zu sein, nicht auf. Ohne Rücksicht auf persönliche Nachteile kämpfte er im Vormärz überall dort mit, wo es um die Errichtung einer Schweizer Demokratie ging. Dazu gab es im aufgeschlossenen Kanton Bern und besonders im damaligen Biel viele Möglichkeiten.

4. BECKERS POLITISCH-AGITATORISCHE TÄTIGKEIT IN BIEL UND UMGEBUNG BIS ZUM JAHRE I 847

\section{a. Seine Stellung zu sozialen und politischen Problemen um ${ }_{1} 840 / 42$}

Wenn wir Beckers Meinung, die Meinung eines am Anfang der dreißiger Jahre stehenden emigrierten deutschen Handwerkers, auf sozialem und außenpolitischem Gebiete näher kennenlernen wollen, so sind es besonders zwei Schriften, die uns einen Einblick in seine Gedankenwelt um diese Zeit gestatten. Seine Ansichten zur sozialen Frage finden sich in einem Brief Beckers vom Januar 1842 an Simon Schmid in Lausanne. ${ }^{2}$ In diesem Brief schreibt Becker, daß er sich zum Gütergemeinschaftlertum, wie es Wilhelm Weitling vertritt, nicht bekennen könne, obwohl er (Becker) einer sozialen Reform von ganzer Seele zugetan sei.

„Begnügen wir uns doch, erst das zu erlangen, was sich nach dem Wesen unserer Zeit, dem Charakter unseres Geschlechts und dessen Forderungen und Bedürfnissen nach, erlangen läßt... Vor Jahren war ich eurer Theorie ganz zugetan aber das Leben und die Erfahrungen haben mich eines anderen belehrt." 3

In einer heute sehr seltenen Schrift: „Ein Wort über die Fragen der Zeit" 4 nimmt Becker I84I zu dem politischen Weltgeschehen Stellung. Es kann nicht meine Aufgabe hier sein, auf diese von

1 Die Vorträge der Direktion der Justiz und Polizei, Mappe Januar I 847, Nr. 674, Herrn Joh. Phil. Beckers Naturalisationsgesuch. (Beckers Naturalisation wird für das Land als nützlich erachtet.) In: Staatsarchiv Bern.

2 J. Ph. Becker, Brief aus Biel vom 29.1.1842 an Simon Schmid in Lausanne, in: WeitlingPapiere, P. 239. I. Fasz. f. Nr. 5, in: Staatsarchiv Zürich. Für die Úbersendung einer Kopie dieses Briefes bin ich Herrn Dr. U. Helfenstein vom Staatsarchiv Zürich sehr verbunden.

${ }^{3}$ Ebenda.

${ }^{4}$ J. Ph. Becker, Ein Wort..., a.a.O. 
Dr. Wirth eingeleitete Schrift näher einzugehen. Ich möchte nur darauf hinweisen, daß Becker in dieser Abhandlung besonders drei Themenkreise umreißt: das Verhältnis Deutschlands zu Frankreich, die orientalische Frage, die Frage Krieg oder Frieden. Gleichzeitig fordert Becker immer wieder, die Völkergleichheit zu sanktionieren, die unterdrückten Völker zu befreien, und sowohl das Frankreich des Bürgerkönigs als auch das zaristische Rußland an Eroberungszügen zu hindern. ${ }^{1}$ Die Abneigung Beckers gegen jede absolute Herrschaft, sein dauerndes Eintreten für die Freiheit - „Darum Freiheit vor allem, das Übrige von selbst", so hatte er sich in einer kleinen Broschüre später geäußert ${ }^{2}$ - führten ihn bald in die demokratischen Bestrebungen des Schweizer Vormärz.

\section{b. Seine Teilnabme an den Freischarenzügen gegen Lu₹ern}

Eine unmittelbare Folge der Jesuitenberufung nach Luzern waren die Freischarenzüge. Eine kleine Gruppe freisinniger Luzerner war über die Entscheidung des Rates so erbost, daß sie sich zu einer Schilderhebung entschloß. Gemeinsam mit den Gesinnungsgenossen anderer Kantone wollte man am 8.12.I 844 losschlagen. Zu denen, die sich über die Jesuitenberufung empörten, gehörte auch Becker, der bei seiner Tätigkeit als Handelsreisender, ,während der er auf die politische Wühlerei" ${ }^{3}$ mehr Zeit als auf seine Geschäfte verwandte, oft die schönen Gestade des Vierwaldstättersees bereist hatte und auch nach Luzern gekommen war. Dort traf er häufig mit Luzerner Freisinnigen zusammen. Nachdem Becker seine Geschäfte mit dem Schwanenwirt erledigt hatte, begab er sich in die "Leuengrube". Dort hörte er die Herren Winkler, Bühler von Büron u.a., wie sie sich über das Aufkommen der Reaktion in der Schweiz ereiferten. Wenn Becker diesen Männern zuhörte, wie sie ganz fest umrissene Taten forderten, so hätte er selbst am liebsten gleich mit zugeschlagen. Während in der "Leuengrube" das liberale Element vorherrschte, versammelten sich im „Raben” die Vertreter der Arbeitervereine. Sie vertraten eine revolutionäre Richtung. $\mathrm{Da}$ Becker sowohl die liberalen als auch die radikaleren Kreise Luzerns gut kannte, gewann er einen guten Úberblick über die Verhältnisse in der Stadt zur Zeit der Jesuitenberufung 1844 .

1 Ebenda, S. 3 I.

${ }^{2}$ J. Ph. Becker, Die Neutralität nach dem Märchen vom Menschenfresser, exzähIt von Joh. Phil. Becker. Zum Frommen der Menschheit. Den gesetzgebenden Versammlungen aller Völker, vornehmlich aber der Schweizerischen Tagsatzung gewidmet, Biel I 848, S.8. ${ }^{3}$ J. Ph. Becker, Aus meinen Erinnerungen, in: Der Republikaner, Volkskalender auf das Jahr I 878, hrg. von R. Rüegg, Winterthur 1878 , S. 66. 
Als man in Biel am 8.12.1844 davon hörte, daß es in Luzern zu einer Entscheidung kommen sollte, wurde am Vormittag in aller Eile eine Versammlung im Rathaus einberufen, die beraten sollte, was zu tun sei. Ungefähr 70 Personen trafen dort zusammen. Es kam zu einer lebhaften Diskussion. Becker gehörte wie sein Freund Ernst Schüler zu denen, die im Sinne der Aufmunterung zur Teilnahme am Waffengang sprachen. ${ }^{1}$

Eine Bürgerwehr wurde organisiert, die auch die Anordnungen zur Aufstellung eines Freikorps treffen sollte. Dieses Freikorps hatte sich dem Landsturm gegen Luzern anzuschließen. Die Begeisterung dieser Bieler Freischar, der auch Becker angehörte, war so groß, daß man am 9.I2.I844, als die Nachricht von Solothurn kam, die Liberalen seien in Luzern schon besiegt, doch noch um 16 Uhr im Gasthaus "Jura" in Biel eine Versammlung durchführte und gegen 17 Uhr die Schar abzog. ${ }^{2}$ Becker berichtet, daß er sich an die Spitze des Bieler Fähnleins stellte. ${ }^{3}$ An irgendwelchen Kampfhandlungen nahm diese Freischar nicht teil. Als man in Solothurn erneut von der Niederlage der Luzerner hörte, kehrte man in aller Stille nach Biel zurück. ${ }^{4}$

Wenn auch dieser erste Freischarenzug gegen die Jesuiten in Luzern fehlschlug, bezw. das von Becker geführte Bieler Fähnlein überhaupt nicht zum Einsatz kam, so hatte Beckers Teilnahme meiner Meinung nach deshalb eine ziemliche Bedeutung, weil er sich damit schon 1844 offen zum Antijesuitismus bekannte. Damit bezog er aber vom Anfang an eine klare Stellung in einer Frage, die nicht nur für den Kanton Luzern, sondern für die Entwicklung der Eidgenossenschaft als Lebensfrage angesehen werden mußte. ${ }^{5}$

Die Wut über die Jesuiten war in der Schweiz so groß, daß bald wieder Versammlungen stattfanden, in denen über einen neuen Freischarenzug gesprochen wurde. Als in der Nacht vom 30. zum 31.3.1845 zwei Kolonnen von Huttwyl und Zofingen gegen Luzern aufbrachen, schlossen sich diesem Zuge, den Ulrich Ochsenbein führte, auch so Bieler Bürger ${ }^{6}$, unter ihnen Johann Philipp Becker, an. ${ }^{7}$

1 Albert Maag, Oberst Johann Philipp Becker von Biel und die deutsch-helvetische Legion 1848-1849 in: Der kleine Bund, literarische Beilage des „Bund" vom 14.10.1928, Jg. 9, Nr. 42, S. 329-33I.

${ }^{2} \mathrm{G}$. Blösch, Chronik von Biel von den ältesten Zeiten bis zu Ende I 873, Biel 1875, S. 227.

3 J. Ph. Becker, Curriculum vitae... a.a.O., in: Archiv für..., a.a.O., S. 3 I6.

4 G. Blösch, Chronik von Biel..., a.a.O., S. 227.

5 Hans Spreng, Ulrich Ochsenbein, Bern I918, S. 31.

${ }^{6}$ G. Blösch, Chronik von Biel..., a.a.O., S. 228; vergl. auch: Die Sceländer im Freischarenzug von 1845 (Vortrag im Historischen Verein von Biel 27.3.1908, in: Bieler Neujahrsblatt 1909, S. 9-29 (Becker nicht erwähnt).

7 J. Ph. Becker, Curriculum vitae..., a.a.O., S. 316. 
Bekanntlich kam es bei Malters zu einem furchtbaren Gemetzel. Die Freischaren wurden vollkommen besiegt. Wie groß die Anteilnahme Biels an den Ereignissen war, geht aus der Bieler Chronik deutlich hervor. Man umlagerte am 2.4.I845 scharenweise den Postwagen, um die ersten Nachrichten zu hören. Der $\mathrm{Ha} B$ der Bevölkerung richtete sich bald gegen die Regierung, die den Zug zuerst gebilligt hatte und, wie man glaubte, in letzter Minute den Freischärlern in den Rücken gefallen wäre.

In einer Stadt wie Biel, wo man sich so entschieden für die Freischarenzüge bekannte, gewann Becker, der sich an beiden Zügen beteiligt und bei der Propagierung derselben mitgewirkt hatte, immer mehr an Ansehen. Wenn auch mancher vom Standpunkt der formalen Staatsordnung aus die Freischarenzüge verurteilte, weil er in dem bewaffneten Uberfall auf einen anderen Kanton eine grobe Rechtswidrigkeit sah, so gab es doch sehr viele gute Patrioten, die den Freischärlern schon deshalb nicht böse sein konnten, wiel diese mit den Waffen für ein Ziel kämpften, das die Mehrheit des Schweizer Bürgertums erstrebte: für die geistige Emanzipation gegenüber der kirchlich-politischen Reaktion. ${ }^{1}$

Um Beckers spätere Meinung über die beiden Freischarenzüge zu erfahren, müssen wir in seinen Aufzeichnungen der letzten Jahre blättern. 1877 schreibt er im „Republikaner”:

„Hatte doch gerade das Mißlingen dieser beiden Schilderhebungen den wertvollen Erfolg, dem Schweizervolk seine zeitweilige Aufgabe zu klarerem Bewußtsein zu bringen und es wuchtiger zu gemeinschaftlichen und einheitlichen Bestrebungen anzuregen. Mehr als je sah man jetzt ein, daß nur durch eine radikale Umwälzung einer schweizerisch nationalen, wahrhaft freiheitlichen Entwicklung sichere Bahn geschaffen werden könne, und daß zu diesem Behufe die von der Heiligen Allianz der Schweiz aufgezwungene I8Ijer Bundesakte, welche den Kantonen eine fast absolute Souveränität garantierte und alle Entfaltung gesunden Gemeinlebens verunmöglichte, durch jedwede Mittel beseitigt und demgemäß die Bundesreform auf den Schild gehoben werden müsse... Im Kanton Bern, welcher durch seine Größe und bewaffnete Macht eine maßgebende Stellung in dieser allgemeinen Bewegung einnahm, wurde der Revisionskampf zum Sturze der Regierung um so gründlicher und verbitterter durchgeführt, als eben diese Regierung, welche

1 J. Dierauer, Die Geschichte der Schweizerischen Eidgenossenschaft, Gotha I917, Band 5, S. 679 . 
die Freischarenzüge seinerzeit heimlich begünstigte, nun nach deren Scheitern nur Eselstritte und Verfolgungsmaßregeln für sie hatte." 1

\section{c. Der Wein- und Zigarrenreisende Becker - ein politischer Agitator}

Auf seinen Geschäftsreisen kam Becker in der Mitte der vierziger Jahre des vergangenen Jahrhunderts in fast alle Teile der Schweiz. Er stand mit dem Jungen Deutschland (Marr) in Verbindung, er veranlaßte, daß Petitionen Luzerner Flüchtlinge im Kanton Neuchâtel verteilt wurden ${ }^{2}$, er kam im Gasthof ,Zum Schlüssel” in Bern mit Schweizer Freisinnigen zusammen, unterhielt in Thun enge Beziehungen zu dem freisinnigen Artillerieoffizier Dünz, und er traf sich mit Hans Michel aus Böningen, der damals im Berner Oberland agitatorisch wirkte und von Becker besonders geschätzt wurde. ${ }^{3}$

Als geschickten Erzähler, der über alle politischen Fragen sehr gut orientiert war, sah man Becker überall gern. Seiner gesellschaftlichen Stellung nach gehörte er damals der Bourgeoisie an ${ }^{4}$, seiner Gesinnung nach, wie er selbst schrieb, Gott sei Dank nicht. ${ }^{5}$ Die humorvolle Darstellungsweise und der reiche Schatz schlechter Witze, den er zu bieten hatte, sicherten ihm überall viele dankbare Zuhörer. Wir lächeln oder spotten vielleicht heute darüber. Damals gehörte dies nun einmal zu seiner Tätigkeit als Wein- und Zigarrenhändler. Die Gäste erwarteten von $\mathrm{ihm}$, daß et etwas in dieser Weise zum besten gab. Die politisch-agitatorischen Versammlungen endeten oft mit einem Trinkgelage, das für die patriotischen Anstrengungen, die man aufgewandt hatte, entschädigen sollte. Wie es auf solchen Zusammenkünften vor sich ging, das beschreibt uns Becker sehr ausführlich in seinen Erinnerungen. ${ }^{6}$

An einem Maiabend kam er beispielsweise mit Gesinnungsgenossen in der „Krone” zu Thun zusammen. Zunächst debattierte man, politische Ereignisse standen im Vordergrund. Es ging um die kantonale Verfassungsreform und um die Bundesreform, um Themen also, die so recht geschaffen waren, jedes patriotische Herz höher-

1 J. Ph. Becker, Lustige Fahrt, ein Schwank aus jüngeren Jahren, in: Der Republikaner, Volkskalender auf das Jahr I 877, hrg. von Reinhold Rüegg, Winterthur I877, S. 72.

${ }^{2}$ J. Ph. Becker, Brief vom 5.I.1845 aus Biel an Doeleke [?], in: Série Jeune Allemagne, Procès-verbal contre la propaganda secrète allemande, les clubs de la Jeune Allemagne et la Confédération du Léman, 1845, p. 134, in: Staatsarchiv Neuchâtel. (Für diesen Hinweis bin ich Herrn Staatsarchivar A. Schnegg, Neuchâtel, sehr verbunden.)

3 J. Ph. Becker, Lustige Fahrt..., in: Der Republikaner..., a.a.O., S. 72.

4 Amtsberichte für Biel I $845-47$, in: Staatsarchiv Bern.

5 J. Ph. Becker, Lustige Fahrt... a.a.O., S. 72.

${ }^{6}$ Ebenda, S. 74 . 
schlagen zu lassen. Inmitten dieser die Freiheit so hoch achtenden Männer saß der kräftige, blauäugige Pfälzer. Er forderte den Sturz der bestehenden Regierung, die Verstärkung der Bundesgewalt und die Vertreibung der Jesuiten. Bei dieser Gelegenheit nahm Becker aber in der „Krone” auch gleichzeitig die günstige Gelegenheit wahr, die hier versammelten Männer für den Anschluß an die Längendorfer Schützengesellschaft in Solothurn, deren Mitglied er damals schon war, zu gewinnen. ${ }^{1}$ Diese Gesellschaft vereinte in sich revolutionäre Kräfte der Schweiz und stellte sich die Aufgabe, die Bundesverfassung von i 8 is zu stürzen.

Hatte sich dann der "patriotische" Eifer ausgetobt, so ging man dazu über, Bacchus seinen Tribut zu zollen. Becker wurde jetzt wieder ganz Geschäftsmann. Er bot alle seine Taschenspieler-, Bauchredner-, Jongleur-, Akrobaten- und Gesangskünste auf, um seine Freunde $z u$ unterhalten und zum Trinken anzuregen. Wem flösse nicht das Wasser im Munde zusammen, wenn er Beckers eigene Beschreibung liest? Erst kam der bescheidene „La Côte” dann der prickelnde "Yvorne", schließlich wurde tiefroter "Cortaillod" kredenzt. Als der Wirt bemerkte, daß seine Gäste ihren Durst ziemlich gelöscht hatten, ließ er stramme „Hilfstruppen” aufmarschieren. Die pommerschen Gänsebrüste und der westfälische Schinken verursachten von neuem Durst. Jetzt erschien - ich spreche mit Beckers Worten - ,im schlanken Amazonenkleid weltbürgerlicher Rüdesheimer I 834er auf der Arena." 2

Becker gibt ehrlich zu, daß er an solchen Tagen, an denen er das Haus des Wirtes füllte, sowohl sein Gewissen als guter Patriot als auch als Familienvater befriedigen konnte. Dies darf aber keinesfalls zu der irrigen Ansicht verleiten, Becker habe zu jenen „gemütlichen Schoppenstechern" - wie Engels die Pfälzer Revolutionäre von I 849 bezeichnete - gehört, die im Wirtshaus große revolutionäre Pläne entwarfen und dann im Kampfe zuerst aufsteckten. Becker war aus anderem Holz geschnitzt. „Doch sobald es galt, für die Sache Trumpf auszuspielen, wurde auch ohne Bedenken Gut und Blut dafür eingesetzt." 3

Ein klares politisches Programm verfolgte Becker während dieser Zeit nicht. Er war überall mit dabei, wo es darum ging, bestehende reaktionäre Verhältnisse zu beseitigen. In diesem Sinne arbeitete er auch journalistisch. So hatte er nach eigenen Angaben die „Berner Zeitung" mit begründet 4 und war Mitarbeiter der "Jura-Zeitung",

1 Ebenda.

2 Ebenda, S. 75.

${ }^{3}$ Ebenda, S. 74.

4. Ph. Becker, Curriculum vitae..., a.a.O., S. 316. 
die sich die Verbreitung patriotischer Grundsätze zum Ziel gesetzt hatte und in entschiedener Opposition zu der lauen Berner Regierung und den anderen Kantonsregierungen stand. ${ }^{1}$

\section{BECKERS FREIHEITSREDE AUF DEM LÄNGENDORFER FREISCHIEBEN IN BIEL AM I 5.8 .1847}

Becker hatte die Bedeutung der Schweizer Schützenvereine für die politische Entwicklung der Schweizer Bevölkerung schon sehr früh erkannt und sich deshalb schon seit Beginn der vierziger Jahre als Mitglied verschiedenster Schützenvereine betätigt. ${ }^{2}$

Die eidgenössischen Schützenfeste waren oft Zentren politischer Agitation. Alle Versuche, die Politik von den Schützenfesten fernzuhalten, waren fehlgeschlagen. Anläßlich dieser Veranstaltungen traten die Vertreter der verschiedensten politischen Richtungen auf, um ihre Gedanken zu entwickeln. Männer wie Baumgartner, Munzinger oder der sehr geschätzte Oberst Dufour sprachen über brennende politische Tagesprobleme. ${ }^{3}$ Besonders aber benutzten die Radikalen die Schützenfeste als eine Tribüne für ihre politische Agitation. ${ }^{4}$

$\mathrm{Da}$ Becker bei solchen Gelegenheiten oft und gern das Wort ergriff, soll hier Beckers Tätigkeit auf dem großen Schützenfest in Biel im August I 847 gewürdigt werden. Er wirkte damals als Präsident des Längendorfer Zweigvereins in Biel. ${ }^{5}$ Unter seiner Leitung hatten sich viele tatkräftige Männer in Biel vereint, um hier eine sehr wichtige Frage: Krieg oder Frieden mit dem Sonderbund, zu beraten. ${ }^{6}$ Aus der Chronik von Biel und aus den Tageszeitungen können wir die Vorbereitungsarbeiten und den Verlauf des Festes entnehmen.? Das sogenannte Längendorfer Freischießen begann am r 5.8.1847. Schützenbataillone nahmen die Straßen vom Hotel du Jura bis

${ }^{1}$ Ebenda, S. 3 I6. - Becker arbeitete noch 1848 als Mitarbeiter für die Jura-Zeitung. So veröffentlichte er „Erinnerungen eines Freiwilligen der Berner-Reservedivision.” Dies ist ein Teil des Berichts, der sich in Ochsenbeins NachlaB (siehe später) befindet. -Die Jura-Zeitung, Biel vom 16.19. und 20.1.1848.

J. Ph. Becker, Curriculum vitae..., a.a.O., S. 316.

3 Theodor Curti, Geschichte der Schweiz im 19. Jahrhundert, Neuenburg 0.J., S. 470.

4 . Flückiger, Freisinnige Politik in Biel seit roo Jahren, in: Bieler Jahrbuch 1929, III. Jahrgang, Biel I 929, S. 37.

${ }^{5}$ Das Komitee des Längendorfer Schießens in Biel (I $5 .-18.8 .1847$ ) bestand aus den Herren Becker, Schüler, von Greyerz, Nickles, Schilling, M. Michel, R. Müller, A. Ritter, Gerichtspräsident Bützberger, Major Funk u.a., in: Die Jura-Zeitung, Biel vom 17.8. 1847, Nr. 98; Der Seeländer Anzeiger, 18.8.1847, Nr. 33 .

6. Ph. Becker, Curriculum vitae..., a.a.O., S. 3 I6.

7 G. Blösch, Chronik von Biel..., a.a.O., S. 232, siehe auch: Der Seeländer Anzeiger, Bern, vom I 8.8.1847, Nr. 33; I.9.1847, Nr. 35; Die Jura-Zeitung, Bern, vom 5.8.1847, Nr. 93 ; 17.8.1847, Nr. 98 ; und: Manual des Regierungsrates, Nr. 125, Schreiben vom 24. Juni 1847 , in: Staatsarchiv Bern. 
jenseits Bözingen ein. Gegen to Uhr wurde die Ankuft der Längendorfer Schützen in Bözingen angekündigt. Der Zug setzte sich darauf nach dem Schützenplatz in Bewegung. An 20000 Menschen waren in Biel auf den Beinen. ${ }^{1}$ J. Phil. Becker hielt die Eröffnungsrede, die zehnmal von dem donnernden Beifall der Schützen unterbrochen wurde. ${ }^{2}$

\author{
„Freunde! Eidgenossen, Waffenbrüder!
}

Es ist mir der schöne Auftrag geworden, Euch alle, alle herzlich willkommen zu heißen; ich soll Euch einführen in den Geist unseres Festes, soll Euch einführen in die Herzen Eurer Schützenbrüder, in die Hütten warmer Gastfreundschaft.

Wohlan! Ihr kennt den Geist, der aller Männer Brust beseelt und in der Liebe wohnt für Menschenglück; den Geist, der jede Tugend nährt und, wenn es ruft, das Vaterland, die treuen Söhne führt in's blutge Schlachtgewühl, den Geist, der, wo ein Volk von ihm durchdrungen, die Berge ebnet und die Meere trocken legt; den Geist, der kühn zusammenwirft die hohlen Staatsgerüste und aus den Trümmern eine neue Schöpfung ruft. Ja, dieser Geist, der soll der Geist von unserm Feste sein. Auf seinen Flügeln werde es getragen; er heb' mit Adlerschwung es hoch empor! Er segne uns und unser Fest!

Die Zeit ist ernst und groß, sie ist verhängnisvoll: Allgemeine Fragen der Kultur, spezielle zur Entwicklung der Nationen fordern unabweisbar ihre Lösung. Schon ist schwül die Luft, schon wettert's dumpf am Himmel; die schwachen Herzen zittern, die kleinen Seelen winseln schon; doch hoch jauchzt auf der Mann als Mann; je mehr die Blitze zucken und die Donner krachen. Was unabweisbar ist im Gang der Weltgeschichte, das wird geheiligt durch die Tat, wird glücklich nur durch sie vollbracht.

Nur im Streben nach Freibeit, nur im Kampfe für die Freiheit, genießt, erwirbt und konserviert man die Freiheit. Nur im ungestörten Wachstum kann erblühn ihr herrlicher Baum und kann er uns spenden seine goldenen Früchte. In den äußeren Spitzen seiner Zweige kreisen seine edelsten Säfte. Könnt Ihr Euch vorstellen einen Baum, der keine Blätter treiben darf, keine Blüten kann entfalten und doch die schönsten Früchte tragen soll. Seht, Freunde, so wäre beschaffen der unmögliche Baum des überweisen Konservatismus.

Vor diesem Wurme wollen wir bewahren das Werk unseres Baumes, damit seine Blätter nicht fallen, seine Äste nicht dorren und sein Stamm nicht verfault, denn er soll aufwachsen hoch und himmelan!

'Unverzagt' und 'Vorwärts' heißt unser 'Scheibenstich', unverzagt und vorwärts soll unseres Festes Stichwort sein! Vorwärts geht des Menschen erster Schritt; vorwärts geht der Völker erste Bahn, vorwärts drängt mit Allgewalt die ganze Natur. Und auf der Völker Vorwärtswege ist kein Berg zu hoch, keine Kluft zu tief, kein Strom zu reißend und kein Meer zu groß.

Und jedes Volk hat ein unveräußerliches Recht, ein Urrecht, damit auch eine heilige Pflicht: Alles, alles aus dem Wege zu räumen, was ihm hinderlich ist auf seinem Entwicklungsgange. Dieses Recht ist älter als alle Verträge der Welt, älter als die Heilige Allianz, älter als der Fünfzehnerbund, älter als das Freischarengesetz. Es ist so alt, als die Schöpfung und so jung, wie der morgende Tag.

Und wenn die Völker vorwärts wollen in friedlicher Entwicklung, wer wälzt ihnen heutzutage die großen Steine in den Weg? Die Jesuiten. Und wie und wo konnte diese Lügenbrut sich einnisten in unserm frei und treu genannten Schweizerlande?

In den Falten der Fünfzehnerbundesakte, diesem Zeugnis fremder Anmaßung und inneren Hochverrats. Und wie heißt der große Stein, an dem wir Schweizer nun zunächst anstoßen? Der heißt Sonderbund.

1 Ebenda.

2 Die Jura-Zeitung, Biel, 17.8.1847, Nr. $9^{8}$. 
Und ich sage Euch: Solange dieser Stein nicht zu Staub geschlagen, solange die Jesuiten nicht in die Hölle gejagt, die Bundesakte nicht auflodert in den Flammen eines Nationalverfassungsfeuers, solange haben wir unser Recht nicht geübt und unsere Pflicht nicht erfüllt, solange haben wir keinen Frieden verdient. Darum unverzagt und vorwärts!

Aber wer soll helfen, was kann retten? Die Behörden. Leider muß derselben guter Wille ersticken in der Zwangsjacke abgestorbener Formen. Nur das Volk, nur es, durch vereinte Kraft, nur ein Volksbund, kann retten. Nur das Volk ist frei und wird nicht tatlos bleiben an den Ufern des Rubicon, es wird nicht unergriffen das Vaterland versinken sehen, sondern mutig sich werfen in die wogende Flut.

Und ist der Sonderbund die Leibjacke der Jesuiten, so müssen die Längendorfer und alle patriotischen Schützen eine Schutzwache des Volksbundes bilden.

Wer unter uns wäre so kurzsichtig und so feige, eine fremde Einmischung zu fürchten. Siehen wir allein in der Welt?

Haben nicht alle Völker ein einziges Ziel? Fragt sie doch über den Alpen, über dem Juta und über dem Rhein; laßt sic doch abstimmen über unsete Unabhängigkeit- und Freiheitsbestrebungen und über die Politik ihrer Kabinette. Die Zeit der Kabinettskriege ist vorbei. In dem Augenblicke, wo die Regierungen Noten wechseln, schreiben sich die Völker Liebesbriefe. Darum unverzagt und vorwärts.

Und die Schweiz? Wir dürfen es uns gestehen, sie steht jetzt an der Spitze der geistigen Bewegung unseres Weltteils Europa: Europa blickt auf sie, Europa wartet auf sie; sie hat eine $\mathrm{Kraft}$, die nie unterliegt, sie hat ein Schwert, es ist unbesiegt. An Europa eine Proklamation zur rechten Zeit und die Tyrannen sollen wagen den Schuß gegen die Schweiz; sie sollen versuchen den Kampf gegen die Zivilisation: Der erste fremde Kanonendonncr wäre das Signal zu einer europäischen Revolution. Die Schweiz ist kein Krakau und kein Portugal, sie ist das Herz Europas, sie ist der Kern der alten Welt; und Europa läßt sich sein Herz nicht verwunden und der ergibt sich nicht!

Inzwischen gehen wir unseren Weg unverzagt und vorwärts, recht tuend und niemand scheuend; und wenn die Stunde schlägt, so stehen wir auf der Bresche und fegen sauber unseren Herd; da wollen wir erneuern der Väter Heldenruhm, da wollen wir vollbringen, was wir unter festlichem Jubel anderwärts geschworen und hier noch geloben werden.

Ist es auch nicht so lieblich der Freiheit Hoch im Schlachtgewühl, als bei des Trinkspruchs Becherklang, so ist es doch des Mannes höchste Wonne, sein schönster Hochgenuß.

Und wenn, o wackrer Schweizermann! die Gattin tränenvoll Deinen Arm ergreift und sich die lieben Kindlein klemmen an Dein Vaterherz, wenn laut und lauter Dir des Gatten und des Vaters Pflicht erwacht, dann zage nicht!

Hinaus zum Kampfe!

Fort in der Brüder Reihen! Und wenn die Erde bebet im Kanonendonner, die Kartätschen unsere Glieder lichten: Freiheit lebe!

Und wenn der Herzfreund fällt, der liebe Bruder sinket an der Seite, die Freibeit lebe!

Und wenn verwundet unter tausend Leichen die Roß und Troß noch übers Leben schreiten: die Freiheit lebe!

Und wenn man Dir der Feinde Sieg verkündet, und wenn die Berge wanken und die Alpen stürzen, Dein letzter Atemzug, Dein letztes Wort: die Freiheit lebe! Das Vaterland.

Doch noch ein Wort, ich muß es sagen, es steht in aller Schweizermänner Brust geschrieben:

Die Feinde siegen nicht, die Alpen stürzen nicht, die Schweiz ergibt sich nicht!

Ewig lebt das Vaterland, ewig lebt die Freiheit, hoch und ewig hoch!" 1

Beckers Rede gipfelte in der Forderung nach Freiheit. Um aber diese Freiheit zu erringen, muß der überweise Konservatismus überwunden

${ }^{1}$ Beckers Rede ist abgedruckt in: Die Jura-Zeitung, Biel, 17.8.1847. 
werden, müssen die Jesuiten ihren Einfluß verlieren, muß der Sonderbund durch den Volksbund patriotischer Schweizer ersetzt werden.

Das Fest wurde bis tief in die Nacht hinein gefeiert. Die illuminierte Stadt bot einen prächtigen Anblick. Verschiedene Musikkorps sorgten für Unterhaltung. Als die Schützen aus Bern verabschiedet wurden, ergriff Becker erneut das Wort:

„Chutzen und Chutzenaspiranten!

Es gibt mancherlei Vögel, gute und schlimme. Unser Vogel ist der Chutz, auf den wir und die Freunde des Vaterlandes jetzt mit Vergnügen sehen. Aber auch unter uns Chutzen gibt es einen Unterschied, es gibt da alte und junge, der alte Chutz ist nicht immer den Jahren nach der alte, aber er hat seine Jugendkraft verloren, seine Feggen werden lahm, er will nicht mehr weit und lustig fliegen. Die jungen Chutzen dagegen kennen keine Schranken, ihre Kraft ist groß, und so weit ihre Kraft reicht, so weit wollen sie fliegen.

Sie sind das ewig junge Menschengeschlecht, das da ringt und kämpft und Schranken durchbrechen will, welche Dummheit und Bosheit um dasselbe gezogen. Und da sind sie in ihrem guten Rechte, denn des Menschen Geist, des Menschen Kraft, des Menschen göttlicher Funke ist es, was ihm Schranken setzt. Jede andere Schranke ist vom Unrecht und muß eingerissen und zertrümmert werden. Chutzen und Chutzenaspiranten!

Ihr versteht mich, wir verstehen uns! So ziehet denn heim zu den Eurigen, und haltet traue Wacht, und wenn die Glocke schallt und das Feuer lodert von den Bergen herab, dana die Waffe zur Hand und ins Glied getreten. Lebet wohl!" 1

Auch diesmal dankten die Schützen wieder mit einem donnernden Hoch. ${ }^{2} \mathrm{Daß}$ ein Fest wie dieses bei der aufgeschlossenen Bevölkerung Biels ansprach, ist selbstverständlich. Organisation und Festverlauf waren wesentlich mit von Becker bestimmt worden. Ist es da verwunderlich, daß er sich innerhalb der Bevölkerung Biels mehr und mehr Anerkennung erwarb? Selbst der Seeländer Anzeiger von Bern sprach Becker ein Lob aus:

„Gehaltreich, zur republikanischen Tugend, zur Freiheits- und Vaterlandsliebe entflammend war die Eröffnungsrede des wackeren Herrn Becker. Schade nur, daß noch immer so viele Leute sind, die hie und da schwanken und sich auf die Höhe der ausgesprochenen Grundsätze noch nicht zu schwingen vermögen.” 3

$\mathrm{Da}$ das Schützenfest von Biel als eine machtvolle Demonstration gegen den Sonderbund angesehen wurde, gewann Becker darüber hinaus auch in den politischen Kreisen der Schweiz, die den Kampf gegen den katholischen Einfluß und damit gegen den Sonderbund führten, Ansehen.

${ }^{1}$ Die Jura-Zeitung, Biel, I9.8.1847, Nr. 99, Titelseite.

2 Ebenda. Diese Reden mögen uns heute ein Lächeln abnötigen; für die damalige Zeit waren sie jedoch charakteristisch. Da Becker viele solcher Reden hielt, wurden diese beiden hier im Wortlaut wiedergegeben.

3 Der Seeländer Anzeiger, 18.8.1847, Nr. 33. 
6. BECKERS TEILNAHME AM SONDERBUNDKRIEG IN DER BERNER RESERVEDIVISION UNTER OCHSENBEINS FÜHRUNG IM NOV. I 847

Beckers Teilnahme am Sonderbundkrieg stellt den Höhepunkt seiner vormärzlichen Tätigkeit in der Schweiz dar und zeigt ihn uns erstmals an verantwortlicher leitender Stelle. Ich darf kurz auf die bekannten Tatsachen hinweisen.

Die Ursachen des Sonderbundkrieges sind im Wiener Kongreß zu suchen. ${ }^{1}$ Nachdem am 4.II.I847 die Tagsatzung den Beschluß gefaßt hatte, militärisch vorzugehen, wurde Dufour Oberbefehlshaber der gegen den Sonderbund eingesetzten eidgenössischen Armee. ${ }^{2}$ Dufour teilte seine Truppen in 6 Divisionen ein. Dazu kam noch die Reservedivision unter dem Befehl Ochsenbeins. Dieser Reservedivision gehörte auch Johann Philipp Becker an. ${ }^{3}$ Die militärische Tätigkeit Beckers in der Division, die bisher in Einzelheiten nicht bekannt war, konnte ich einem von Becker verfaßten Bericht an den Kommandanten der bernischen Reservedivision, Ochsenbein, entnehmen. ${ }^{4}$

1 Werner Näf, Der Schweizerische Sonderbundskrieg als Vorspiel der deutschen Revolution von 1848 , in: Basler Zeitschrift für Geschichte und Altertumskunde, Basel I92 I, Band XIX, Heft I, S. 1o.

${ }^{2}$ J. Dierauer, Geschichte der..., a.a.O., Band 5, S. 734-735.

${ }^{3}$ J. Ph. Becker, Curriculum vitac..., a.a.O., S. 316.

$4 \mathrm{~J}$. Ph. Becker, Bericht an den Kommandanten der bern. Reservedivision Herm Oberst Ochsenbein von Joh. Phil. Becker (Adjutant Ochsenbeins) in Biel. Mit einer Karte des Kampfplatzes der Gefechte bei Schüpfheim, den 22./23 Nov. I847), I I I Seiten (handschriftlich von Becker). Der Bericht enthält folgendes Inhaltsverzeichnis:

Seite:

Seite:

Feldzug nach Freiburg . . . . I Das zweite Gefecht bei Schüpfheim . 47

Einmarsch der Avantgarde in den

Kanton Freiburg . . . . . . . 3

Besetzung des Dorfes Bösingen . . 5

Einmarsch der Hauptkolonne . . 7

Besetzung des Dorfes Düdingen . . 9

Der erste Kriegsgefangene . . . . II

Unsere Soldaten teilen ihren Mund-

vorrat mit den Kriegsgefangenen . I3

Das Feldlager bei Düdingen ... . I5

Die Armee auf dem Rückmarsch . . 20

Der Feldzug nach Luzern . . . . 25

Das Gefecht bei Escholzmatt . . . 26

Das erste Gefecht bei Schüpfheim . 29

Das Feldlager bei Schüpfheim . . . $4_{4} \mathbf{I}$

Marsch von Schüpfheim nach

Entlebuch ........ 64

Umgehung der Position Bramegg . 77

Die Armee auf der Bramegg . . . . 84

Marsch der Armee gegen Luzern und einer Kolonne über Schwarzenberg 85

Unsere Truppen retten mit Lebensgefahr dic Möbel eines brennenden Hauses . . . . . . . . . 87

Hauptquartier in Kriens . . . . . 93

Marsch über Luzern nach Sursee . 98

Marsch über Zell nach Langenthal . 100

Hauptquartier in Kirchberg . . . 103

Einzug und festlicher Empfang in

Bern .......... Ios

Besuch der Verwandten und

Abschied ....... . IO8-I I I

Der Bericht Beckers befindet sich in: Nachlaß von Ulrich Ochsenbein, Sonderbundkrieg, Akten der Berner Reservedivision, Mappe 2, in: Staatsarchiv Bern. 
Dieser zum Teil unveröffentlichte Bericht wurde noch nicht entsprechend ausgewertet. Er ist aber für Beckers politische und militärische Entwicklung äußerst wichtig. Becker zeigte nämlich im Sonderbundkrieg schon Ansätze jener militärischen Eigenschaften, die ihn später zu einem der befähigsten Revolutionsgenerale in der badisch-pfälzischen Mairevolution werden ließen. Bekanntlich bezeichnete Friedrich Engels Becker als den einzigen deutschen „Revolutionsgeneral". 1

Becker nahm als eidgenössischer Stabssekretär ${ }^{2}$ und Adjudant Ochsenbeins am Sonderbundkrieg teil.

Beckers ausführlicher Bericht an Ochsenbein, dessen Inhaltsverzeichnis ich auf S. 272, Anm. 4, mit angegeben habe, beginnt wie folgt:

„Ihrem Wunsche gemäß will ich es hiermit versuchen, alles, was ich in beiden Feldzügen der bernischen Reserve Mitteilungswertes wahrgenommen, möglichst umständlich $\mathrm{zu}$ berichten. Ich werde mich zwar hierbei hauptsächlich an das halten, was Ihnen in taktischer und strategischer Beziehung sowie in Beurteilung militärischer Eigenschaften von Offizieren und in Abrundung des Geschichtlichen von Wichtigkeit sein mag." 3

Am I 2.I I.r 847 früh 4 Uhr erfolgte der Abmarsch der Division von Bern. ${ }^{4}$ Bis zur Ankunft in Laupen ereignete sich nichts Bemerkenswertes. Becker erforschte die Stimmung der Soldaten und stellte fest, daß man überall bei der Truppe kriegslustig und heiter war. Mit der Avantgarde rückte Becker in den Kanton Freiburg ein und gab beim Durchwaten der Sense schon ein gutes Beispiel persönlicher Einsatzbereitschaft. ${ }^{5}$ Mit dem Lager von Düdingen ${ }^{6}$, das so ganz nach dem Geschmacke Beckers war, schloß die erste Etappe des Feldzuges siegreich ab und die Division begab sich wieder nach Bern.?

1 F. Engels, Dem Gedächtnis..., a.a.O., in: Der Sozialdemokrat..., Titelseite.

${ }^{2}$ J. Ph. Becker, Bericht an den Herrn Platzkommandanten Oberst Funk in Bern, Bern I I.1 1.1847, in: Nachlaß Ochsenbeins, Akten der Berner Reservedivision unter Oberst Ochsenbein, 1847, Nov. I.-15., Mappe I, in: Staatsarchiv Bern. Becker unterschreibt hier als eidg. Stabssekretär. - Siehe auch: Militärdirektion, Manual, Band 2 vom 2.8.31.1 2.1847, Seite 238. In einem Schreiben vom 5.11.1847 zeigt der Chef des Stabes die Brevetierung Beckers als Stabssekretär an. In Staatsarchiv Bern.

3 J. Ph. Becker, Bericht an den Kommandanten..., a.a.O., S. I.

1 Ebenda, S. I.

${ }^{5}$ Ebenda, S. 3.

- Ebenda, S. 8.

7 Ebenda, S. 2 I-22. 
Dann begann am I6.11.1847 der Marsch gegen Luzern. Gegen Io Uhr brach man von Bern in Richtung Emmenthal auf. ${ }^{1}$ Nachdem Becker mit dem Stab in Sumiswald Quartier bezogen hatte, erhielt die Division am 2 I.I I.I847 von General Dufour den Befehl, bei Trubschachen am 22. II.1847 in den Kanton Luzern einzumarschieren. Mit der Avantgarde rückte Becker gegen Escholzmatt vor, wo man den Feind mit wenigen Schüssen vertrieb. ${ }^{2}$

Besonders bei den beiden Gefechten in der Nähe Schüpfheims zeichnete sich Becker aus. Am 22.11.1847 is Uhr begann das erste Gefecht bei Schüptheim. ${ }^{3}$ Becker hatte mit dem Kommandanten Brugger für eine ordentliche Truppenaufstellung gesorgt. Die Soldaten zeigten teilweise wenig Angriffslust. Becker ermunterte sie immer wieder zum Vorrücken. ${ }^{4}$

$\mathrm{Da}$ die Kampfhandlungen nicht den gewünschten Verlauf nahmen, befahl Ochsenbein Becker, dafür zu sorgen, daß besonders auf der Höhe 2 alle Befehle ordnungsgemäß ausgeführt würden. Die führenden Offiziere, besonders Hauptmann Moser aus Herzogenbuchsee, sahen Beckers Kommen gar nicht gern. Als Becker die Soldaten aufforderte und ein Offizier bemerkte, daß Becker ihnen nichts zu befehlen habe, sprang der Pfälzer auf ihn ein, drohte, ihn mit dem Säbel in Stücke zu schlagen, wenn man nicht vorrückte. ${ }^{5}$

Becker wollte unbedingt das vom Feind besetzte Kloster und die feindliche Kanone erbeuten. Er war in diesem Kampfe so draufgängerisch, daß die eigenen Soldaten ihn zurückhalten wollten und ihn auf die persönliche Gefahr, in die er sich begab, aufmerksam machten.

Gegen Mittag, am 23.11.1847, war das zweite Gefecht zu Schüpfheim beendet, sicher das bedeutendste, an dem Becker im Sonderbundkrieg teilnahm. Den Marsch ins Entlebuch machte Becker in der Plänklerlinie des rechten Flügels der Avantgarde mit ${ }^{6}$, mit der er auch in Hasli einrückte und dort das Terrain erkundete.

Ochsenbein hatte im Verlaufe des Feldzuges Becker als einen jederzeit einsatzbereiten Offizier kennengelernt. Damit mag es auch zusammenhängen, daß der Divisionsgeneral am 24. I I. 847 bei Tagesanbruch Becker zu sich rufen ließ und ihm den Befehl erteilte, mit zwei Scharfschützen- und 4 Füsilierkompagnien die feindliche

\footnotetext{
1 Ebenda, S. $22 \mathrm{ff}$.

2 Ebenda, S. 26.

3 Ebenda, S. 29.

4 Ebenda, S. 30.

5 Ebenda, S. 54.

B Ebenda, S. 64.
} 
Position Bramegg zu umgehen, anzugreifen und tüchtig zu manövrieren. ${ }^{1}$

Becker war über den Auftrag sehr erfreut, bedauerte später nur, daß der Feind sich schon zurückgezogen hatte und so kein Einsatz erfolgte. ${ }^{2}$

Der Kampf gegen Luzern wurde zugunsten der Antijesuiten entschieden. In Kriens, wo Becker mit Ochsenbein und dem Stabe wieder zusammentraf, gab man sich den Siegesfreuden hin. Über Sursee, Langenthal, Kirchberg ging es dann in den nächsten Tagen nach Bern zurück, wo am 29.I I.I 847 unter dem Jubel der Berner Bevölkerung den Truppen von Regierungsrat Funk der Dank ausgesprochen wurde. ${ }^{3}$

Im „Bären” wurde das Siegesfest gefeiert. Am nächsten Tage mußte sich Becker mit schwerem Kopf daran machen, einen Bericht über das Verhalten des Hauptmanns Moser bei Schüpfheim abzufassen. ${ }^{4}$

Schon am I.I 2.1847 suchte Becker Ochsenbein in der Tagsatzung auf, wo letzterer wieder als Bundespräsident den Vorsitz führte, und bat wegen dringlicher häuslicher Geschäfte um seine Entlassung. Obwohl eine vielköpfige Familie auf ihn wartete, er viele Handelsund Fabrikgeschäfte zu erledigen hatte, tat Becker der Abschied sehr weh. ${ }^{5}$

Seinen Bericht an Ochsenbein beendete er mit den Worten:

„Die Reservearmee ist nicht ohne schätzbare Eroberung heimgekehrt in den Schoß des bürgerlichen Lebens, sie hat den schönen unveräußerlichen Schatz treuer Pflichterfüllung mit nach Hause gebracht, und wenn dem wackeren Reservemann in späteren Jahren die Zeitumstände und vergängliche Lebenskraft neue Taten versagen, so greift er in die Vorratskammer der Erinnerung und lebt von dem eroberten Schatze einer tatenvolleren Vergangenheit." 6

Zum Schluß drückt Becker noch den Wunsch aus, daß dem Vater-

1 Ebenda, S. 74.

2 Ebenda, S. $77-80$.

Ebenda, S. 107.

4 J. Ph. Becker, Bericht an den Kommandanten der bernischen Reservedivision Herrn Oberst Ochsenbein, I.I 2.1847, in: Ochsenbeins Nachlaß, Mappe 2 unter Akten, die Rechtfertigung des Scharfschützenhauptmanns Moser betreffend, I847 Nov./Dez. Siehe auch: Schreiben Mosers an Ochsenbein vom 28.1 1.1847, in: Staatsarchiv Bern.

${ }^{5} \mathrm{~J}$. Ph. Becker, Bericht an den Kommandanten..., a.a.O..., S. I Io.

6 Ebenda, S. I IO-III. 
lande der Frieden erhalten bleiben möge, er aber im Falle der Gefahr bereit sei, wieder unter Ochsenbeins Kommando zu kämpfen. ${ }^{1}$

\section{ZUSAMMENFASSUNG}

Ich hoffe, daß es mir gelungen ist zu zeigen, daß der Pfälzer Johann Philipp Becker, der 1838 in die Schweiz emigrierte, während der ersten zehn Jahre, bis 1847 , sich tatkräftig für die demokratische Schweizer Freiheitsbewegung einsetzte. Ich glaube, daß vor allem seine Rede in Biel 1847 und seine Teilnahme am Sonderbundkrieg diese Feststellung erhärten, eine Feststellung, die auch von der zeitgenössischen Presse unterstützt wurde, als man Becker später angriff:

„Überall, wo es in den Kämpfen der jüngsten Zeit Mühe und Opfer erheischte, da war er keiner der letzten. In dem Sonderbundkrieg hat er, so sehr es ihm seine Geschäftsstellung erschwerte, nebst seinem Sohne freiwillig in den Reihen der Eidgenossen gestanden. Wie er sich dabei gehalten, so kann dem Solothurner Blatt der Chef der Bernischen Reservedivision, so wie die ganze Mannschaft sagen." 2

Ich habe bewußt das Jahr I 847 als Abschluß dieser Darstellung genommen, weil Beckers politisches Wirken bis dahin im wesentlichen Schweizer Interessen diente. Seine Tätigkeit I848/49 in Biel, als er die Stadt zu einem revolutionären Zentrum in der Schweiz machte, seine Stellung als Leiter des Zentralausschusses der Deutschen in der Schweiz, später als Präsident des Wehrbundes „Hilf Dir” zielte auf die Errichtung einer deutschen Republik. ${ }^{3}$ Die Darstellung dieser Zeit möge einer weiteren Abhandlung vorbehalten bleiben.

1 Ebenda, S. Ir 1 .

2 Die Jura-Zeitung vom i9. Juli 1848 , Nr. 169.

${ }^{3}$ Georg Trübner, Johann Philipp Becker - ein Leben für die Freiheit (1809-1886). (Unter Berücksichtung aller von Becker verfaBten Schriften und Zeitungen, seines Nachlasses in Amsterdam und anderen unveröffentlichten Quellenmaterials, besonders aus Schweizer Archiven.) Phil. Habil. Jena I957, 2 Bände, hier Band I (Maschinenschrift) 